
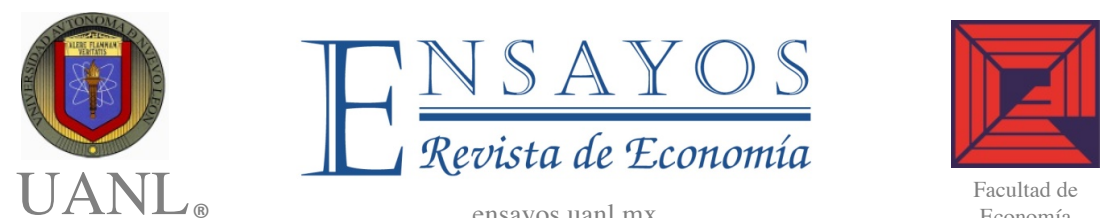

\title{
Demanda de electricidad residencial: Una perspectiva de regresión cuantílica
}

\author{
Residential electricity demand: A quantile regression \\ perspective
}

Ricardo Arturo Cárdenas Ovalle*

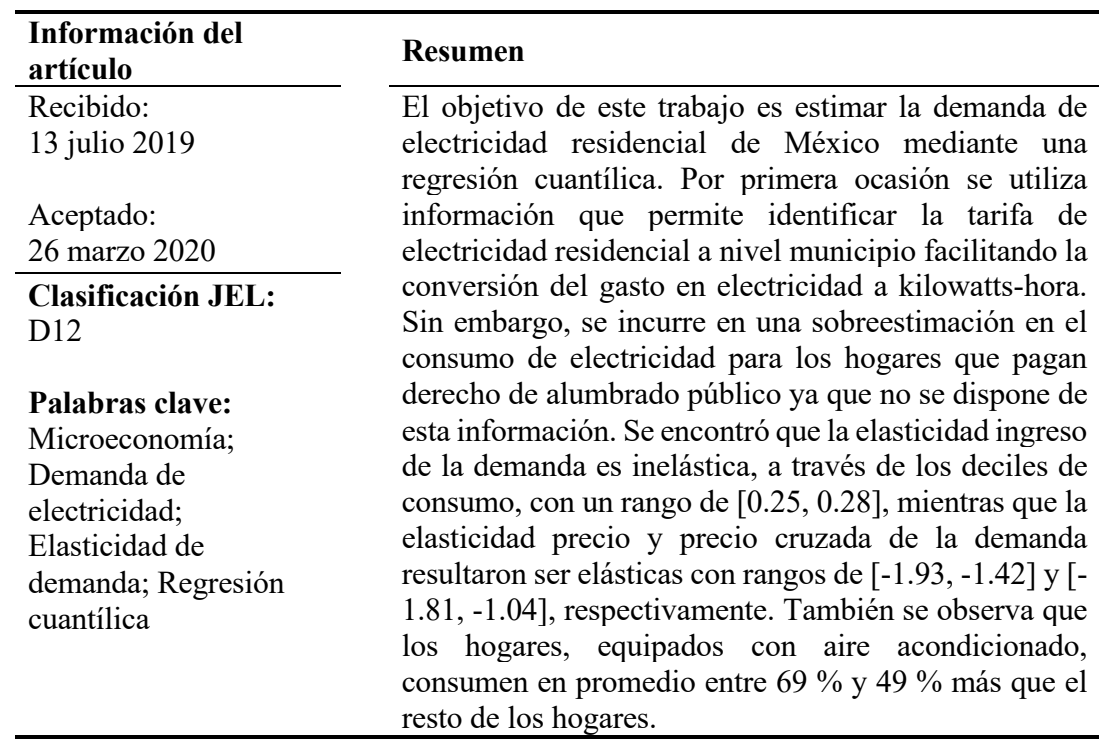

*Banco de México, email: ricardo.arturo.ovalle@gmail.com.

Nota: Los anexos pueden ser consultados en http://dx.doi.org/10.29105/ensayos39.1-4 ISSN Electrónico: 2448-8402 | ISSN Impreso: 1870-221X | C2020 Los autores @) () 


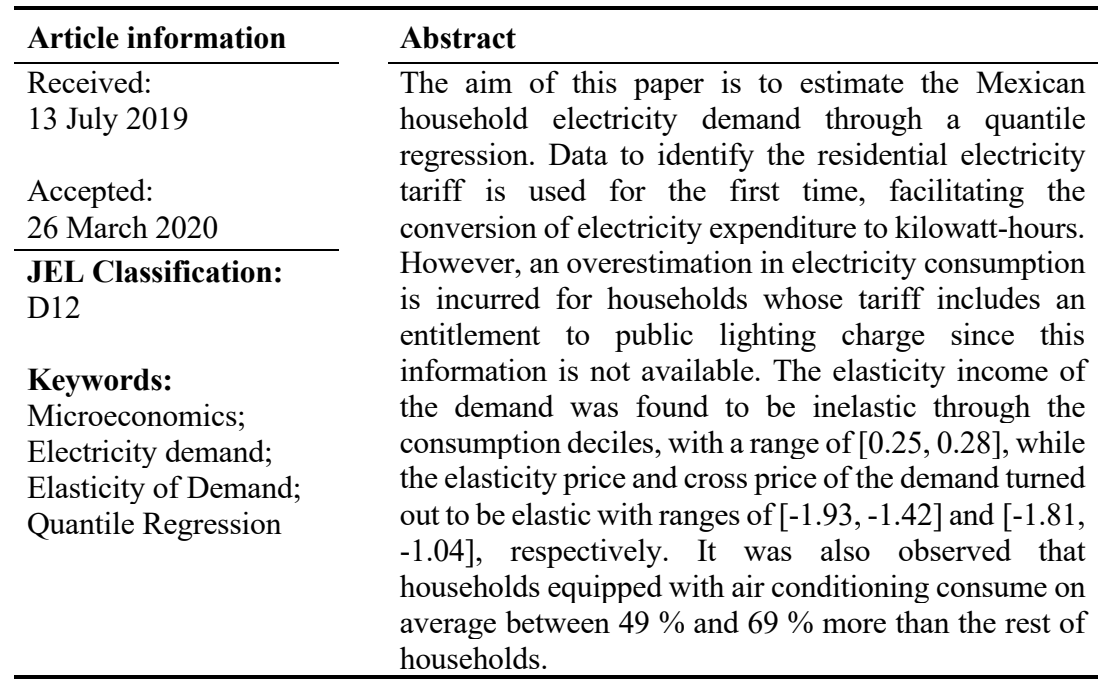

\section{Introducción}

El estudio de la demanda de electricidad con frecuencia es abordado mediante datos agregados debido a la falta de información que permita conocer el consumo de kwh de los hogares. Otros estudios recurren a la información de las encuestas para estudiar la demanda de electricidad ya sea recuperando la información de la cantidad consumida de electricidad por medio del gasto en electricidad, como lo hace Fernández (2006), o limitándose al estudio del gasto en electricidad, que es el caso de Medina y Vicéns (2011). También existen algunas encuestas que capturan la información del consumo de electricidad medido en kilowatts-hora (kwh), un ejemplo de esto es el trabajo de Branch $(1993)^{1}$.

Con el estudio de los determinantes del consumo de electricidad es posible identificar sus efectos ante ajustes en la política pública. Cuando estos efectos son significativos, resulta de interés conocer sus correspondientes magnitudes y direcciones con la finalidad de corregir patrones de consumo de electricidad no deseados, ya que los hogares podrían estar haciendo un uso ineficiente de la electricidad mediante un consumo de electricidad que exceda el nivel de consumo eficiente cuando con menores niveles de consumo se podría obtener el mismo nivel de bienestar. El consumo eficiente de electricidad debe ser aquel que es obtenido como resultado de la asignación eficiente del equipamiento y consumo de energía utilizando correctamente toda la información. Por lo que, aun siendo este nivel de consumo alto, podría

\footnotetext{
${ }^{1}$ A partir de ahora, de manera indistinta se entenderá consumo de kwh, consumo de electricidad o simplemente consumo como la cantidad de electricidad consumida.
} 
considerarse racional siempre que cumpla con los requisitos listados, pero cuando el precio de la electricidad no refleja el verdadero valor para el cual el mercado se encuentra en equilibrio y este está por debajo de este, los hogares no tienen incentivos de alterar su asignación de consumo en electricidad.

En México, los subsidios a la electricidad incentivan a los hogares en hacer un mal uso del recurso, y esto a su vez deriva en asignaciones ineficientes en el equipamiento de la vivienda, ya que los hogares no solo consideran el precio de los componentes del equipamiento de la vivienda, sino que también consideran el precio de la energía que requieren para su función.

Un análisis empírico contribuye en la detección de patrones de consumo ya que, mediante técnicas cuantitativas, se pueden identificar los determinantes del consumo de electricidad y sus efectos sobre esta (magnitud y dirección). En este estudio se ha realizado un análisis exhaustivo sobre la función de demanda de electricidad residencial, este incluye un esfuerzo por estimar la cantidad consumida de kwh de los hogares considerando su nivel de gasto y precio kwh, es decir que se hace un estudio riguroso de la dinámica de precios de electricidad en el sector residencial mediante la revisión de contratos de suministro de energía eléctrica, permitiendo así identificar la estructura tarifaria que enfrentan los hogares. Se utiliza la información de la Encuesta Nacional de Ingresos y Gastos de los Hogares partiendo del gasto en electricidad, declarado por los hogares en la encuesta, para obtener el consumo de kwh y a su vez un precio medio por kwh. Con lo anterior, es posible construir una función de demanda de electricidad residencial que considera las variaciones en ingreso, precio y otros factores económicos y sociales que determinan el consumo de electricidad. Esto resulta útil para los tomadores de decisiones en la implementación de políticas públicas del sector energético, los cuales formulan dichas políticas en función de objetivos, en ocasiones olvidando que los instrumentos de políticas públicas son medios y no fines ${ }^{2}$.

\footnotetext{
${ }^{2}$ Los instrumentos son utilizados por los hacedores de políticas públicas, que enfrentan decisiones del tipo "trade-off" (utilidad a cambio de sacrificio) en la búsqueda de sus objetivos fijados. Los instrumentos de política económica se pueden distinguir por su dinámica de interrelación, siendo incompatibles (conflictos de carácter fundamental), complementarios o independientes. Esta dinámica de objetivos puede presentarse por las disyuntivas entre objetivos económicos (eficiencia), financieros (sostenibilidad y rentabilidad financiera), sociales (acceso, equidad y asequibilidad), etc., cuando estas se contraponen. Así, el objetivo social de asequibilidad puede ser, hasta cierto punto, opuesto al de eficiencia económica y compatible hasta otro (Cuadrado, 2010). Por ejemplo, si el objetivo de eficiencia económica nos lleva a un resultado de reducción de costos, entonces las tarifas podrían ser más asequibles contribuyendo así al objetivo social, pero también la tarifa podría ser muy baja y no compensar los costos de producción y de inversión que garanticen el servicio de electricidad en el largo plazo, los objetivos serían incompatibles. Entre los instrumentos se pueden distinguir los cuantitativos, cualitativos, y reformas fundamentales (Tinbergen, 1987). Las políticas cuantitativas son ajustes en el nivel de los
} 
El objetivo de este trabajo es analizar los determinantes de la demanda de electricidad residencial de México. La Encuesta Nacional de Ingresos y Gastos de los Hogares (ENIGH) únicamente captura el gasto en electricidad así que se realiza una conversión del gasto en electricidad a cantidad consumida de electricidad. Por primera ocasión se utiliza información de la Comisión Reguladora de Energía (CRE) que permite identificar la tarifa de electricidad residencial a nivel municipio y proporciona el periodo de temporada de verano para cada municipio. La demanda de electricidad residencial se estima mediante un enfoque de regresión cuantílica por ser más robusta en la estimación de modelos multivariantes cuando se trabaja con información proveniente de encuestas con tamaños muestrales grandes y ante la presencia de heteroscedasticidad, observaciones atípicas o cambio estructural (Vicéns y Sánchez, 2012). Adicionalmente se incorpora al análisis información de los precios medios de electricidad, precios máximos de gas licuado de petróleo y una aproximación de la temperatura a nivel municipal.

El presente artículo se organiza de la siguiente manera: en la primera sección se realiza una revisión de la literatura; en la segunda se expone la metodología y en la tercera se presentan resultados y conclusiones.

A continuación, se hace mención de algunos trabajos en los que estiman la demanda de electricidad de los hogares, se comienza haciendo una revisión general de los estudios previos y se termina con una breve mención de aquellos que utilizan un enfoque de regresión cuantílica.

\section{Antecedentes}

Los trabajos que se exponen en esta sección tienen como principal objetivo la estimación de las elasticidades ingreso, precio y precio de la demanda de electricidad. Un ejemplo es el trabajo de Branch (1993), en el que se estima una función de demanda de electricidad con datos de panel para Estados Unidos, utilizando la información de la de la encuesta "Consumer Expenditure Survey" (CE). La unidad de observación de la encuesta son los hogares y la población objetivo del estudio son los hogares en que residen los propietarios, ya que los servicios públicos están incluidos en el pago de la renta. Branch utiliza el método de Mínimos Cuadrados Generalizados (MCG) ya que el estimador de Mínimos Cuadrados Ordinarios (MCO) resultaría ineficiente debido a la correlación del error a lo largo de los meses dentro de cada hogar en la muestra. El autor estima la elasticidad ingreso y precio de la demanda en 0.23 y -0.20 , respectivamente.

instrumentos ya disponibles en la economía (ajustes de tarifas, bloques de consumo, frecuencia de cobro del servicio, etc.); las políticas cualitativas son inserciones de cambios estructurales en la economía (cambios en la ley, introducción de impuestos, regulaciones); y las políticas de reformas fundamentales influyen sobre la base de la organización socioeconómica (políticas de ordenación). 
Por otro lado, Filippini y Pachauri (2004) estiman tres ecuaciones de demanda de electricidad residencial mediante MCO, con información de la encuesta "National Sample Survey Organization" (NSSO), para las temporadas de invierno, verano y monzón en zonas urbanas de la India. Los autores obtienen elasticidades ingreso de la demanda similares entre temporadas, en un rango de 0.60 a 0.64 , mientras que la elasticidad precio de la demanda resulta ser más inelástica durante el verano, con -0.29 , que en invierno y monzón donde obtuvieron -0.42 y -0.51 , respectivamente. También estiman la elasticidad precio cruzada de la demanda con el precio del gas LP y obtienen que esta es menos inelástica durante la temporada de monzón, con -0.65 , que en las temporadas de invierno y verano que resultaron en -0.27 y 0.26 , respectivamente.

Asimismo, Fernández (2006) estima la demanda de electricidad de los consumidores domésticos en España durante 1999 estudiando las variaciones de la cantidad consumida de electricidad, la cual obtiene a partir de la conversión del gasto en electricidad reportado por los hogares en la Encuesta Continua de Presupuesto Familiares (ECPF) ${ }^{3}$. La autora utiliza un enfoque de MCO recurriendo a la técnica de variables instrumentales para controlar la endogeneidad entre el precio y cantidad consumida de kwh. El problema de endogeneidad surge por un sistema de bloques de consumo el cual consiste en un incremento de precio por bloque de consumo. Finalmente, estima una elasticidad ingreso de la demanda de entre 0.14 y 0.58 , mientras que para la elasticidad precio de la demanda obtiene $-0.59^{4}$.

Igualmente, Agostini, Plottier y Saavedra (2012) estiman la demanda de electricidad residencial en Chile con información de la Encuesta de Caracterización Socioeconómica Nacional (CASEN), durante 2006, con un enfoque de MCO. Los autores obtienen elasticidades ingreso, precio y precio cruzada de la demanda de entre $[0.11,0.12],[-0.38,-0.40]$ y $[0.14,0.16]$, respectivamente ${ }^{5}$. Finalmente, señalan que si bien la elasticidad ingreso es inelástica, la elasticidad precio resulta menos inelástica de forma que un cambio en precio tiene mayor impacto en el consumo de electricidad que un cambio en ingreso.

\footnotetext{
${ }^{3}$ Fernández (2006) utiliza una factura estándar, que había sido contratada por el $90 \%$ de la población, para realizar la conversión del gasto en electricidad a cantidad de electricidad consumida.

${ }^{4}$ El rango para la elasticidad ingreso de la demanda de Fernández (2006) son las estimaciones puntuales mínima y máxima obtenidas por la inclusión de variables ficticias por decil de ingreso.

${ }^{5}$ La elasticidad precio cruzada de la demanda es en referencia al precio del gas LP.
} 
He y Reiner (2016) a través de datos panel provenientes de una encuesta dirigida por la Universidad de Pekín, la cual es representativa para Beijing, Shanghai, y Guangdong, estiman la demanda de electricidad para hogares de Beijing con el objetivo de identificar un rango de necesidades básicas en el consumo de electricidad. Al igual que Branch (1993) los autores estiman el modelo mediante MCG, para las zonas urbana y rural, y encuentran un umbral de consumo eléctrico con respecto a los ingresos de cada zona la cual estiman en $90 \mathrm{kwh}$ para zonas rurales y $150 \mathrm{kwh}$ para zonas urbanas. Los autores incorporan variables ficticias por decil de ingreso, pero solo resultan significativas a partir del séptimo decil para zonas rurales con valores entre 0.34 y 0.39 , y a partir del quinto decil de ingreso para zonas urbanas con un rango de entre 0.33 y 0.46 . Por otro lado, las elasticidades precio resultaron en -3.59 para zonas rurales y -2.91 para zonas urbanas, mientras que las elasticidades precio cruzada de la demanda resultaron en -2.03 para zonas rurales y -1.36 para zonas urbanas. De esta forma, las elasticidades precio y precio cruzada de la demanda, a diferencia de otros estudios, resultaron ser elásticas.

La distribución del consumo de electricidad es asimétrica por la presencia de observaciones extremas. Al igual que la media aritmética no es siempre el estimador de tendencia central más representativo, el efecto marginal medio que ofrece el estimador de MCO no es siempre el más representativo ante la presencia de observaciones atípicas, heteroscedasticidad o cambio estructural (Vicéns y Sánchez, 2012). Una alternativa al enfoque de MCO es el enfoque de regresión cuantílica el cual ha sido utilizado recurrentemente en otros trabajos para la estimación de la demanda de electricidad ya que permite obtener líneas de regresión por cuantil aprovechando todas las observaciones de la muestra. Entre los artículos que utilizan esta técnica de regresión se encuentra el trabajo de Medina y Vicéns (2011) en el que se estima la demanda de electricidad residencial en España a través del gasto en electricidad de la ECPF. Los autores realizan la estimación para el primer, segundo y tercer cuartil, al igual que la estimación de $\mathrm{MCO}$, obteniendo elasticidades ingreso de la demanda entre 0.12 y 0.15 en las estimaciones por cuartiles y 0.16 con el estimador de MCO. Adicionalmente, los autores presentan una segunda estimación en la cual utilizan variables ficticias por nivel de ingreso para estimar las elasticidades ingreso de la demanda. Finalmente concluyen que las variaciones en el ingreso disponible tienen un bajo impacto en las variaciones del consumo de electricidad.

Igualmente, con el objetivo de identificar características socioeconómicas, geográficas o del equipamiento del hogar, relacionadas con altos niveles de ineficiencia en el consumo de electricidad, Hancevik y Navajas (2015) realizan una estimación de la demanda de electricidad residencial, para el área metropolitana de Buenos Aires, mediante un enfoque de regresión cuantílica. 
Los autores obtienen el consumo de kwh a partir del gasto en consumo de electricidad de la Encuesta Nacional de Gastos de los Hogares (ENGH) y del cálculo de la factura de electricidad residencial. La estimación de la elasticidad ingreso de la demanda resulta entre 0.23 y 0.26 , mientras que la elasticidad precio cruzada de la demanda resulta tener poca relevancia, obteniendo estimaciones entre -0.18 y 0.26 . Los autores no estiman la elasticidad precio de la demanda ya que argumentan escasa variación en el precio. Entre los resultados de este estudio se encuentra que los hogares que no tienen acceso al servicio de gas natural en promedio consumen más electricidad que los que sí tienen, que los hogares con equipos de aire acondicionado tienen en promedio mayores niveles de consumo que los que no tienen y que los hogares que habitan en departamentos en promedio consumen menos electricidad que los que habitan en casas.

Para el caso de México se encuentra el trabajo de Gutiérrez (2016) que estima la demanda de electricidad con un enfoque de regresión cuantílica utilizando los datos de la Encuesta Nacional de Ingresos y Gastos de los Hogares (ENIGH) 2014. Las conclusiones principales del estudio son que las características socioeconómicas no resultan ser relevantes en la determinación del consumo de electricidad y, como en otros estudios, la elasticidad ingreso de la demanda tampoco resulta ser importante. También encuentra que los hogares que están equipados con aire acondicionado tienen un consumo promedio mayor a los que no lo tienen y que la temperatura de las entidades federativas influía de manera importante en el consumo de electricidad.

A pesar de que en este trabajo, al igual que el de Gutiérrez (2016), se utiliza información de la ENIGH 2014, se consideran ciertos aspectos que proporcionan un valor agregado a la investigación, entre los cuales se encuentran: 1. Se cuenta con información de la clasificación tarifaria a nivel municipal, por lo anterior no es necesario realizar asignaciones, que podrían ser distintas, a la de la metodología de clasificación tarifaria de $\mathrm{CFE}^{6} ; 2$. Se cuenta con variabilidad por clasificación tarifaria a nivel municipal y por bloque de consumo de electricidad; 3 . Se considera que el impuesto de derecho de alumbrado público (DAP) no se cobra en todo el país y no se determina de la misma forma para todas las localidades (Nuño, 2014), por lo que a falta de información se evita descontar dicho impuesto en el gasto de electricidad de los hogares asumiendo el costo de esta limitación, ya que se considera que un ajuste arbitrario podría introducir un sesgo mayor en la determinación del

\footnotetext{
${ }^{6} \mathrm{La}$ CFE asigna tarifas de electricidad residencial distintas a la 01 a las localidades que durante tres o más de los últimos cinco años excedan, con su temperatura media mínima anual, una temperatura establecida para cada tarifa. La información anterior se obtiene consultando para cada tarifa, particularmente para el año 2014, en los enlaces de "Tarifas específicas domésticas: 1A, 1B, 1C, 1D, 1E y 1F” dentro de la página web de CFE.
} 
consumo de kwh de los hogares que el asumido al no hacerlo; 4. Se aprovecha otra información contenida en la encuesta para identificar consumos realizados dentro y fuera de la temporada de verano (fecha en que se realizó el pago y frecuencia de pago) así como la identificación de usuarios de $\mathrm{CFE}^{7} ; 5$. Se considera la variable precio de electricidad en el modelo de forma que se evita la subespecificación del modelo de demanda por omisión de esta variable relevante.

Dado las observaciones anteriores, y al hecho de que el presente trabajo cuenta con información que permite asociar las tarifas y temporadas de verano con los hogares de la ENIGH 2014 (información que no se encuentra disponible para cruzar con la ENIGH 2016), se justifican la realización de la estimación de la demanda de electricidad para México con información de esta encuesta ${ }^{8}$.

\section{Metodología}

Esta sección se divide en cuatro partes: En la primera se expone el modelo teórico; en la segunda se presenta el modelo empírico; en la tercera se describe el modelo econométrico a utilizar; y en la cuarta se muestra la estimación del modelo.

\subsection{Modelo teórico}

La demanda de electricidad se deriva de una demanda de servicios que sean provistos mediante el uso de aparatos eléctricos, por lo que la acumulación de electrodomésticos y la intensidad con que estos se utilizan determinan el consumo de electricidad. La intensidad del uso de aparatos eléctricos está en función del ingreso, precio de la electricidad, equipamiento del hogar, características demográficas, temporada y clima (Branch, 1993). Por tanto, la demanda de electricidad se modela de la siguiente forma:

$$
Q_{k w h}=f\left(I, P_{k w h}, H, E, D, T, C\right)
$$

donde $Q_{k w h}$ es la cantidad consumida de electricidad, $I$ el ingreso del hogar, $P_{k w h}$ el precio de la electricidad, $H$ características de la vivienda, $E$ el equipamiento del hogar, $D$ las características demográficas, $T$ la temporada y $C$ el clima.

\footnotetext{
7 Ver en "Manual de Disposiciones Relativas al Suministro y Venta de Energía Eléctrica Destinada al Servicio Público', sección segunda, décimo séptima.

URL: $<$ http://www.cfe.gob.mx/inversionistas/InformacionJuridica/Paginas/Manuales.aspx> [Último acceso: Agosto de 2017].

${ }^{8} \mathrm{La}$ información de las tarifas es obtenida a través de la calculadora de facturas de electricidad de la CRE: URL < http://www.calculadora.cre.gob.mx> [último acceso: agosto de 2017].
} 


\subsection{Modelo empírico}

Partiendo del modelo teórico de Branch (1993) se utilizan como variables explicativas el ingreso, precio de electricidad y el precio del gas LP $\left(P_{G_{L P}}\right)$ para estimar la elasticidad ingreso, precio y precio cruzada de la demanda, respectivamente. Igualmente, se considera el tamaño del hogar, características del jefe del hogar, características y equipamiento de la vivienda, temperatura del municipio y la región en la que se ubica el hogar. No se agrega información sobre la temporada ya que los datos utilizados corresponden a la temporada de verano.

$$
\begin{aligned}
\log \left(Q_{k w h}\right)=\beta_{0} & +\beta_{1} \log (I)+\beta_{2} \log \left(P_{k w h}\right)+\beta_{3} \log \left(P_{G_{L P}}\right) \\
& +\beta_{4} H+\beta_{5} E+\beta_{6} D+\beta_{7} C+v
\end{aligned}
$$

Los datos son obtenidos a partir de la Encuesta Nacional de Ingresos y Gastos de los Hogares (ENIGH) 2014. La motivación principal para utilizar la ENIGH 2014, y no una más reciente, es la obtención de clasificaciones tarifarias de electricidad residencial a nivel municipal para el mismo año de la encuesta, mismas que no están disponibles para el año 2016. Aunque se podría pensar que los municipios pueden tener la misma clasificación tarifaria en el periodo 2014-2016, no existe completa certeza de esto ya que, como se comentó a detalle con anterioridad, estas dependen de los resultados de temperatura media mínima promedio de los últimos cinco años. Igualmente, no se utiliza la información de la ENIGH 2018 ya que esta aún no se publicaba al momento de realizar la investigación.

La ENIGH ofrece información sobre el monto, procedencia y distribución de ingresos y gastos de los hogares; características ocupacionales y sociodemográficas de los integrantes del hogar, así como de la infraestructura de la vivienda y equipamiento del hogar. La encuesta es representativa para México, con corte urbano y rural, así como para las entidades federativas que hayan convenido una ampliación de la muestra con el Instituto Nacional de Estadística y Geografía (INEGI) ${ }^{9}$. A partir del cuestionario de Gastos del Hogar de la ENIGH se obtiene el gasto en electricidad que incluye el pago por servicio de energía eléctrica y el pago proporcional al consumo del hogar cuando comparte el servicio con un negocio al estar dentro de la misma vivienda, y excluye la proporción de gasto en electricidad correspondiente al negocio que se tiene dentro de la vivienda, el servicio de electricidad recibido como pago por trabajo exentando al trabajador del pago y los gastos generados por reinstalación del servicio. El levantamiento de la ENIGH 2014 se realizó entre el 11 de agosto y 28 de noviembre de 2014, de forma que la encuesta

\footnotetext{
${ }^{9}$ La ENIGH 2014 también es representativa para el Estado de Tabasco.
} 
capta el tercer trimestre siendo este el periodo con mayor consumo de electricidad. La ENIGH tiene una muestra de 19,479 hogares, pero solo 17,150 hogares reportaron gasto en energía eléctrica. De estas observaciones únicamente se conservaron 16,577 ya que se eliminaron las observaciones de los hogares que no contaban con gasto monetario en electricidad, que reportaron su consumo de electricidad en un año distinto al 2014 o una frecuencia de pago superior a dos meses, y los hogares que declararon que obtenían la electricidad de una fuente distinta a la del servicio público. Adicionalmente se eliminaron dos observaciones por no contar con información para alguna variable explicativa del modelo.

Se obtiene la cantidad de consumo mensual en electricidad mediante la conversión del gasto en electricidad, una vez descontado el IVA de $16 \%$, utilizando los precios y bloques de consumo de cada tarifa, así como las temporadas de verano ${ }^{10}$. En este trabajo no se utiliza la tarifa de alto consumo (DAC) ya que no se cuenta con el historial de consumo de los últimos doce meses de cada hogar. Igualmente, no se considera el pago por derecho de alumbrado público (DAP) ya que se desconoce el método de cálculo de cobro para cada municipio ${ }^{11}$.

A continuación, se realiza una descripción de las variables explicativas del modelo las cuales pueden provenir de la ENIGH o de fuentes alternas:

a) Elasticidad ingreso, precio y precio cruzada de la demanda

En lugar de utilizar el ingreso como indicador de la renta del hogar se utiliza el gasto no energético ya que el primero puede influir tanto en el consumo de la electricidad como en el equipamiento de electrodomésticos de los hogares (Fernández, 2006), mientras que el gasto no energético indica el poder adquisitivo sin la distorsión sobre la capacidad adquisitiva de equipos eléctricos $^{12}$. El gasto no energético se obtiene sustrayendo el gasto en electricidad y combustibles del gasto total.

\footnotetext{
${ }^{10}$ Además de la ENIGH 2014, se utiliza información sobre los precios de electricidad y bloques de consumo provenientes de la CFE, así como del tipo de tarifa residencial y temporadas de verano por municipio obtenidas de la CRE (ver anexos 1, 2 y 3 ). Las ecuaciones que describen la conversión del gasto en electricidad a cantidad de electricidad consumida se encuentran en el anexo 4.

${ }^{11}$ El DAP no está legislado en todas las entidades federativas de la República Mexicana, y la decisión y mecanismo de cobro puede variar inclusive por colonias dentro de un mismo municipio dependiendo de los acuerdos que tengan las entidades federativas o los municipios con CFE (Nuño, 2014).

${ }^{12}$ Fernandez (2006) señala que esta consideración sobre el ingreso ha sido abordada previamente en Dubin y McFadden (1984), Baker y Blundell (1991), Leth-Petersen (2002) y Halvorsen, Larsen y Nesbakken (2003).
} 
Se utiliza el precio medio de la electricidad ya que para los consumidores es costoso distinguir el precio marginal partiendo de la información proporcionada en su recibo de electricidad (Fernández, 2006). Lo anterior se soporta empíricamente en el trabajo de Shin (1985) en el que se contrasta si los consumidores de electricidad residencial responden al precio medio o al precio marginal, favoreciendo a la hipótesis de que los consumidores responden al precio medio.

Se incorpora el precio del gas LP ya que este podría ser un bien sustituto de la electricidad para algunos servicios como lo son la cocción de alimentos y el calentamiento del hogar. Los precios del gas LP para el usuario final en México se encuentran sujetos a un esquema de precios máximos ya que se considera un bien indispensable para las actividades cotidianas de la mayoría de la población ${ }^{13}$. En el primer decreto por el que se establecieron los precios del gas LP se asignaron 145 zonas con distintos precios máximos los cuales se calculan sumando el precio de venta de primera mano; flete del centro embarcador a la planta de almacenamiento para distribución; margen de comercialización; e impuesto al valor agregado. Para incorporar la variable precio del gas LP se utilizó tanto la información de los precios máximos del gas LP provenientes del SIE durante el año 2014, como el primer decreto en el que se establecen los municipios que integran cada zona ${ }^{14}{ }^{15}$.

\section{b) Características de la vivienda}

Para indicar las características de la vivienda se incorporan las variables tamaño y tipo de vivienda. Para el tamaño se utiliza la proporción de bombillas sobre número de cuartos, bajo el razonamiento de que cuartos grandes requieren mayor iluminación. Mientras que para el tipo de vivienda se incorporan variables ficticias de casa, departamento, vecindad, cuarto en azotea y local no construido para habitación.

\section{c) Equipamiento de la vivienda}

Respecto al equipamiento del hogar, se considera si este cuenta con calefacción, aire acondicionado, acceso a gas, bombillas ahorradoras y una variable ficticia que indica si el hogar cuenta con medidor de luz ${ }^{16}$. También

\footnotetext{
${ }^{13}$ A partir del 01 de enero de 2017 se liberalizó el precio del gas LP.

${ }^{14}$ Sistema de Información de Energía (SIE). URL: <http://sie.energia.gob.mx>.

${ }^{15}$ El primer decreto, por el que se fija el precio máximo del gas LP, es publicado en el Diario Oficial de la Federación el 28 de diciembre de 2007. URL:

$<$ http://diariooficial.gob.mx> [último acceso: Agosto de 2017].

${ }^{16}$ En la muestra se incluyeron los hogares que declararon que tenían gastos en electricidad y que obtenían la luz eléctrica del servicio público, por tanto, se desconoce la situación de los hogares sin medidor de luz.
} 
se agregan variables ficticias de no acceso a gas y de acceso a gas LP. La ENIGH 2014 no proporciona información directa sobre el acceso a gas de los hogares, pero se deducen bajo los siguientes criterios: 1 . Se considera que los hogares tienen acceso a gas natural si declaran en la encuesta que utilizan gas natural para cocinar o si reportan gasto en gas natural; $y, 2$. Se considera que los hogares tienen acceso a gas LP si declaran que lo utilizan para cocinar, si reportan gasto en gas LP o si la vivienda cuenta con tanque estacionario ${ }^{17}$.

\section{d) Características demográficas}

Para analizar los efectos de las características demográficas se incorpora la zona (rural o urbana) interaccionada con el estrato económico y variables ficticias para cada entidad federativa ${ }^{18}$. Igualmente, se incluyen variables de género, edad y educación del jefe del hogar.

\section{e) Clima}

Para considerar el efecto clima sobre el consumo de electricidad se utiliza la variable de temperatura. La información climatológica por municipio se puede obtener a través de la información generada por la Comisión Nacional del Agua (CONAGUA) ${ }^{19}$, pero no sería viable extraer la información para cada municipio disponible y posteriormente enfrentarse a un problema de asignación ${ }^{20}$. Alternativamente se ha obtenido la temperatura media mensual durante el año 2014 de 190 municipios mediante los 32 anuarios estadísticos y geográficos de las entidades federativas para el año $2015^{21}$. Estas temperaturas se asignan al resto de los municipios bajo un criterio de distancia radial mínima. Se utilizó la información de longitud y latitud de los municipios, para calcular una aproximación de distancia radial, utilizando la formula Haversine,

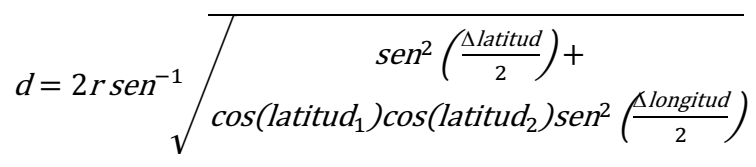

17 Las pregunta 25 y 51 del Cuestionario de Viviendas de la ENIGH 2014 son "El combustible que más usan para cocinar es...1) leña; 2) carbón; 3) gas de tanque; 4) gas natural; 5) electricidad; y 6) otro combustible?" y "Esta vivienda tiene tanque de gas estacionario?". La clave de gasto para gas natural y gas LP en la encuesta son R001 y G009, respectivamente.

${ }^{18}$ En la muestra no se cuenta con observaciones de hogares de estrato medio alto o alto para hogares rurales.

${ }^{19}$ CONAGUA, información climatológica. URL: http://smn.cna.gob.mx.

${ }^{20}$ No hay estaciones meteorológicas para cada municipio por lo que solo se cuentan con algunas mediciones.

${ }^{21}$ En algunos casos no se dispone de información durante el año 2014 por lo que se utilizan los meses del año más próximo 
donde $r=$ radio terrestre ${ }^{22}$.

Esta aproximación de temperatura solo depende de la distancia entre municipios omitiendo otras consideraciones, pero permite mayor variabilidad y es una aproximación más estricta que el promedio de la temperatura por entidad federativa. Además, la temperatura se utiliza únicamente como una variable de control. Para cada hogar se utilizó la temperatura promedio correspondiente a los meses en que realizó consumo de electricidad.

\subsection{Modelo econométrico de regresión cuantílica (Koenker y Basset,1978)}

El método de regresión cuantílica es una alternativa a la de MCO ya que este último es menos robusto por ser sensible ante la presencia de heteroscedasticidad, cambios estructurales y datos atípicos mismos que son frecuentes cuando se trabaja con datos de encuestas. Esta técnica se basa en la minimización de desviaciones absolutas ponderadas con pesos asimétricos los cuales no se ven afectados por datos extremos. A diferencia del estimador de MCO, el estimador de mínimas desviaciones absolutas (LAD), también conocido como mínimos errores absolutos, establece una relación entre los regresores y la variable endógena sin restringir la perturbación aleatoria, por lo que se considera una técnica semiparamétrica. El estimador LAD tiene el inconveniente de limitar el proceso de inferencia ya que al desconocer la distribución del término del error no se pueden analizar las propiedades de los estimadores, aunque a la vez tiene la ventaja de no estar restringido a la normalidad de los residuos (Vicéns y Sánchez, 2012).

Se define cuantil $\theta$ como

$$
\operatorname{Min}(b \in \mathbb{R})\left[\sum_{Y_{i} \geq b} \theta\left|Y_{i}-b\right|+\sum_{Y_{i}<b}(1-\theta)\left|Y_{i}-b\right|\right]
$$

que es un valor que minimiza una suma ponderada, donde se pondera más el segmento con menor número de observaciones. El valor $b$ que minimiza la relación anterior es el de la observación que deja una proporción $\theta$ por debajo de la muestra y otra proporción de $(1-\theta)$ por encima de la muestra.

Los cuantiles incondicionales del valor muestral definidos anteriormente como un problema de optimización también pueden ser tratados como los cuantiles de la variable $Y$ condicionados a los valores de un conjunto de regresores $X$. Si

\footnotetext{
${ }^{22}$ La información de longitud y latitud de los municipios se obtiene del Catálogo único de Claves de áreas Geoestadísticas Estatales, Municipales y Localidades del INEGI. http://www.inegi.org.mx [último acceso: Agosto de 2017].
} 
consideramos que $b$ es una simplificación de $x_{i} \beta$ cuando $x_{i}=1$, entonces podemos expresar el problema de optimización planteado en (4) como

$$
\operatorname{Min}(\beta \in \mathbb{R})\left[\sum_{Y_{i} \geq x_{i} \beta} \theta\left|Y_{i}-x_{i} \beta\right|+\sum_{Y_{i}<x_{i} \beta}(1-\theta)\left|Y_{i}-x_{i} \beta\right|\right]
$$

donde el valor que minimiza la suma cuadrada residual es $\operatorname{Quant}_{\theta}\left(Y_{i} \mid x_{i}\right)=$ $x_{i} \beta_{\theta}$. El estimador de los parámetros que minimiza (5) sería una estimación cuantílica la cual se obtiene normalmente mediante métodos de optimización iterativos o de programación lineal (Medina y Vicéns, 2011). De esta forma se lleva a cabo una minimización de las desviaciones mínimas absolutas donde los residuos positivos tienen ponderaciones diferentes a los residuos negativos.

El método de regresión cuantílica permite estimar rectas de regresión por cuantiles de la variable dependiente sin el inconveniente de ser considerablemente afectada por la presencia de heteroscedasticidad, datos atípicos o cambios estructurales ya que penaliza los errores de manera lineal y no de manera cuadrática como sucede con el estimador de MCO. De esta forma es posible analizar diferentes segmentos de la distribución como los hogares con consumo de electricidad más alto en donde se esperaría encontrar niveles elevados de ineficiencia energética (Hancevic y Navajas, 2015).

Además, se evita el sesgo de selección por segmentación de muestra señalado por Heckman (1979), ya que utiliza las desviaciones respecto a los cuantiles para ponderar la recta de regresión en cada cuantil evitando así la segmentación de la muestra. Aunque al no conocer la distribución del error se limita el proceso de inferencia, al no poder analizar las propiedades de los estimadores, para muestras grandes y bajo ciertas condiciones de regularidad los coeficientes estimados se distribuyen asintóticamente como una normal (Vicéns y Sánchez, 2012). La regresión cuantílica tiene una eficiencia comparable a la de MCO para los modelos lineales gaussianos, mientras que supera sustancialmente al estimador de $\mathrm{MCO}$ en modelos lineales no gaussianos (Koenker y Bassett, 1978). Esta técnica permite analizar a los hogares con diferentes niveles de consumo, en lugar de limitarse al análisis del hogar promedio. La razón para analizar la respuesta de los hogares dependiendo de su nivel de consumo de electricidad es que este puede ser muy alto o muy bajo, de forma que los hogares con consumo alto podrían estar haciendo un uso inadecuado del recurso mientras que los hogares con consumo bajo podrían incrementar su bienestar con pequeños incrementos de consumo de electricidad. Dada la asimetría de la distribución del consumo de electricidad, no es conveniente generalizar las estimaciones arrojadas por el estimador de MCO ya que estas describen al hogar promedio y no necesariamente a los hogares que se encuentran en los extremos de la 
distribución de consumo de electricidad, sobre todo aquellos que se encuentran en el extremo superior a la media de consumo donde la dispersión es mayor. Por tanto, se estudia la respuesta de los hogares a lo largo de la distribución del consumo de kwh obteniendo así información útil para la implementación de políticas públicas en materia de energía eléctrica residencial. Los coeficientes obtenidos en la regresión cuantílica, al igual que el estimador de MCO, corresponden a los efectos marginales en consumo de electricidad. Sin embargo, estos no representan al hogar con consumo de electricidad promedio a menos que el cuantil de consumo de electricidad en cuestión corresponda con el promedio de consumo de electricidad.

\subsection{Estimación del modelo}

El problema de endogeneidad entre las variables consumo de electricidad y el precio de la electricidad se ha encontrado con regularidad en trabajos previos cuando el precio varía por bloques de consumo (Fernández, 2006). Por tanto, estimar el modelo de forma directa no garantiza la consistencia de los estimadores. Se aplicó la prueba de endogeneidad de Haussman para verificar si el problema de endogeneidad, entre el consumo de electricidad y su precio, está presente en el modelo. Se rechaza la hipótesis nula de no endogeneidad para un nivel de $\alpha=0.01$, por tanto, se considera que existe un problema de endogeneidad entre ambas variables ${ }^{23}$. Se utiliza la técnica de variables instrumentales para controlar el efecto que tiene el sistema de incremento de precio por bloques de consumo sobre el precio de la electricidad. Dadas las ecuaciones de precio y consumo de electricidad:

$$
\begin{gathered}
Y_{1}=\gamma_{0}+\gamma_{1} Y_{2}+\gamma_{2} X+\gamma_{3} Z+\varepsilon \\
Y_{2}=\beta_{0 \theta}+\beta_{1 \theta} Y_{1}+\beta_{2 \theta} X+v_{\theta}
\end{gathered}
$$

en donde

$$
\begin{aligned}
& Y_{1}=\log \left(P_{k w h}\right) \\
& Y_{2}=\log \left(Q_{k w h}\right) ;
\end{aligned}
$$

$X=$ Matriz de variables exógenas para $Y_{2}$;

$$
\begin{gathered}
Z=V_{q} * T_{j} ; \\
V_{q}=\left\{\begin{array}{l}
1, \text { temporada de verano } \\
0, \text { de otra forma }
\end{array} ;\right. \\
T_{j}=\left\{\begin{array}{l}
1, \text { tarifas diferentes a la } 01 \\
0, \text { tarifa } 01
\end{array}\right.
\end{gathered}
$$

${ }^{23}$ La prueba de endogeneidad se incluye en el anexo 5. 
Se estima en una primera etapa la ecuación para el precio medio de electricidad por MCO $Y_{1}$ incluyendo todas las variables exógenas $X$ y los instrumentos $Z^{24}$

$$
Y_{1}=\widehat{\Pi}_{0}+\widehat{\Pi}_{1} X+\widehat{\Pi}_{2} Z+\hat{\varepsilon}
$$

y se obtienen los valores estimados

$$
\widehat{Y}_{1}=\widehat{\Pi}_{0}+\widehat{\Pi}_{1} X+\widehat{\Pi}_{2} Z
$$

los cuales se reescriben en la ecuación de precio como la suma entre los valores estimados y los residuos

$$
Y_{1}=\hat{Y}_{1}+\hat{\varepsilon}
$$

misma que se sustituye en la ecuación de consumo de electricidad $Y_{2}$

$$
\begin{aligned}
& Y_{2}=\beta_{0 \theta}+\beta_{1 \theta}\left(\hat{Y}_{1}+\hat{\varepsilon}\right)+\beta_{2 \theta} X+v_{\theta} \\
& Y_{2}=\beta_{0 \theta}+\beta_{1 \theta} \hat{Y}_{1}+\beta_{2 \theta} X+\left(\beta_{1 \theta} \hat{\varepsilon}+v_{\theta}\right) \\
& Y_{2}=\beta_{0 \theta}+\beta_{1 \theta} \hat{Y}_{1}+\beta_{2 \theta} X+v_{\theta}^{*}
\end{aligned}
$$

siendo la (10) el modelo a estimar. A continuación, de manera informativa, se muestran los primeros 9 deciles de consumo de electricidad.

\section{Cuadro 1}

Consumo de electricidad

\begin{tabular}{cccccccccc}
\hline Decil & I & II & III & IV & V & VI & VII & VIII & IX \\
kwh & 53.31 & 78.86 & 100.49 & 120.74 & 144.67 & 176.91 & 221.19 & 281.34 & 446.75
\end{tabular}

Nota: Los valores corresponden al valor del decil y no al promedio de cada decil.

Fuente: Elaboración propia con información de la ENIGH 2014, CFE y CRE.

\section{Resultados}

En este apartado se hace referencia a la significancia estadística a partir de un nivel de $\alpha=0.05$. En el anexo 7 se exhiben las estimaciones con su respectivo nivel de significancia y en el anexo 8 se presentan las estimaciones mediante

\footnotetext{
${ }^{24}$ Bound, Jaeger, y Baker (1995) proponen analizar las características de la primera etapa de la estimación para evitar utilizar instrumentos que estén débilmente correlacionados con la variable endógena ya que podría llevar a la ineficiencia e inconsistencia del estimador. Los autores proponen analizar la $\mathrm{R}^{2}$ parcial y la significancia en esta primera etapa. Los instrumentos utilizados en la ecuación precio resultan significativos al igual que la significancia conjunta del modelo. Por otro lado, se obtiene una $\mathrm{R}^{2}$ de 0.45 y al excluir los instrumentos es de 0.31 , por lo que los instrumentos aumentan la $\mathrm{R}^{2}$ en 0.14 . Véase en anexo 6.
} 
gráficas, mismas a las que se hace alusión en los siguientes párrafos. Las gráficas incluyen las estimaciones de la regresión cuantílica para cada decil de consumo, cada estimación cuenta con su intervalo de confianza y en cada gráfica se incluye el intervalo de confianza de MCO que permite determinar si las estimaciones de la regresión cuantílica son estadísticamente diferentes a las de MCO.

El intercepto corresponde a un hogar cuyo jefe de familia es un hombre menor de 65 años que no ha concluido secundaria y habita en una casa con medidor de luz, con accesos a gas natural, sin calefacción o aire acondicionado, dentro de una población urbana de estrato económico medio, particularmente en la Ciudad de México. El consumo promedio de este hogar se estima en $73 \mathrm{kwh}$ de acuerdo con el estimador de MCO, mientras que para las estimaciones por deciles de la regresión cuantílica resultan entre $15 \mathrm{kwh}$ y $170 \mathrm{kwh}$. La estimación para el intercepto del primer decil de consumo de electricidad no resultó significativa, por lo que no se consideró en el rango anterior. Este resultado se debe tomar con cautela ya que es posible que en la muestra se tengan pocas observaciones, o ninguna, que representen a este hogar hipotético.

Las elasticidades ingreso, precio y precio cruzada de la demanda resultaron en $0.27,-1.73$ y -1.61 , respectivamente, mientras que en las estimaciones por deciles de consumo resultaron entre [0.25, 0.28], [-1.93, -1.42] y [-1.81, -1.04], respectivamente ${ }^{25}$.

En general, la estimación obtenida de la elasticidad ingreso de la demanda es parecida a la de trabajos previos, de forma que los cambios en el ingreso tienen una magnitud pequeña sobre las variaciones en consumo de electricidad. Dado lo anterior, la implementación de una política pública vía ingreso (un incremento en impuestos, por ejemplo), cuyo objetivo sea una mejor racionalización del consumo de electricidad no sería factible. Sin embargo, también supone que cambios en la política fiscal no perturbarían en gran medida la demanda de electricidad residencial. Algo a destacar es que la elasticidad ingreso estimada por Gutiérrez (2016) indica que los hogares con mayor nivel de consumo de electricidad son más sensibles a cambios en ingreso lo cual se atribuye a cuestiones de lujos, mientras que en este trabajo los deciles ocho y nueve de consumo en electricidad resultan ser los menos sensibles ante cambios en ingreso, estas a su vez son las únicas que son estadísticamente distintas a la elasticidad ingreso obtenida por el estimador de MCO (ver gráfica 1). Es importante señalar que existen diferencias metodológicas importantes, entre el estudio de Gutiérrez (2016) y el presente

\footnotetext{
${ }^{25}$ Los rangos de estimaciones reportados, para la regresión cuantílicas por decil, representan las estimaciones puntuales mínima y máxima que además hayan resultado estadísticamente significativas.
} 
estudio, en la determinación de consumo de kwh de los hogares. Además, la elasticidad ingreso de la demanda estimada en este estudio representa a la del hogar, mientras que la de Gutiérrez (2016) representa a la elasticidad ingreso per cápita del hogar.

La elasticidad precio (ver gráfica 2) es mayor en los deciles de consumo altos, además de que algunas estimaciones resultan estadísticamente distintas a la obtenida por el estimador MCO. Es importante destacar que la elasticidad precio de la demanda obtenida es más elástica que la de estudios previos, a excepción de las obtenidas por He y Reiner (2016) que resultan ser más elásticas que las de este trabajo. Las estimaciones sugieren que los hogares son más sensibles ante cambios en precios de electricidad, en comparación a los cambios en ingreso, dicha relación es negativa y en particular mientras mayor es el consumo del hogar en electricidad mayor es su respuesta de disminución de consumo de electricidad ante incrementos en precios. En México el precio medio de kwh pagado por el hogar depende del subsidio que es otorgado en función de la temperatura, es decir que el subsidio no se focaliza por nivel de ingreso y por tanto los hogares que podrían pagar el precio de la electricidad en verano también reciben el subsidio incentivándolos a hacer un mal uso del recurso. Convendría explorar las implicaciones de la determinación de tarifas en México ya que estas podrían estar provocando el uso irracional de electricidad por parte de los hogares.

Por otro lado, la elasticidad precio cruzada de la demanda (ver gráfica 3), al igual que la elasticidad precio, resulta ser más elástica que en otros trabajos, salvo las obtenidas por He y Reiner (2016). Sin embargo, no resultan estadísticamente distintas a la obtenida por el estimador de MCO. Resulta interesante que el consumo de electricidad se reduce ante un aumento en el precio del gas LP, de forma que las estimaciones sugieren que la electricidad y el gas LP son bienes complementarios. El resultado es contrario a lo esperado ya que se pensaría que haya un grado de sustitución entre energéticos, relación encontrada en el trabajo de Agostini, Plottier y Saavedra (2012). Por otro lado, Flippini y Pachauri (2004), utilizando el precio del queroseno, obtuvieron un signo negativo para la elasticidad precio cruzada de la demanda dentro de la temporada de invierno y monzón. Los autores comentan que no esperaban dicho resultado y que para futuras investigaciones se requiere explorar si hay un error en sus estimaciones. En el caso de este estudio, los datos en su mayoría corresponden a la temporada de verano por lo que se podría comparar las estimaciones con las de Flippini y Pachauri (2004) ${ }^{26}$. Sin embargo, el signo

\footnotetext{
${ }^{26}$ La ENIGH se levantó entre el 18 de agosto y 28 de noviembre de 2011 considerando que los consumos en electricidad se realizan durante meses previos, lo cual se puede corroborar con la pregunta “¿cuál fue la fecha del último pago?” contenida en el cuestionario de gastos del hogar.
} 
obtenido por los autores para la temporada de verano, contrario al obtenido en este trabajo, resulta positivo.

La sustitución o complementariedad de los energéticos analizados deben estar en función de su uso. Los hogares utilizan estos energéticos para obtener servicios como lo son cocción de alimentos, iluminación, climatización o acondicionamiento del aire (ventilación, calefacción y refrigeración), lavado, secado, planchado, etc. La flexibilidad con la que los hogares pueden sustituir energéticos dependerá del equipamiento de la vivienda. La cuestión es si los hogares están racionalizando las asignaciones del equipamiento de su vivienda. Los componentes que integran el equipamiento se consideran bienes duraderos y por tanto podrían ser reemplazados hasta que pierdan su funcionalidad o hasta que haya un cambio en los precios de los energéticos tal que incentive a su reemplazo por otros de mayor eficiencia. Se requiere un análisis profundo que explore vertientes como los determinantes de las inversiones de los hogares en el equipamiento de su vivienda, la capacidad de respuesta de los hogares ante cambios en precios de energéticos en sus asignaciones de equipamiento de vivienda, etc. Lo anterior escapa al alcance del estudio por lo que se considera para futuras investigaciones. También, al igual que Flippini y Pachauri (2004), se considera los posibles errores de medición y de muestra, por lo que se sugiere ser cauto con el resultado obtenido.

El tamaño del hogar influye de manera positiva en el consumo de electricidad, aunque los incrementos disminuyen conforme aumenta el número de integrantes en el hogar, de acuerdo con los resultados, en promedio se obtendrían decrementos en el consumo de electricidad a partir del noveno integrante. Esto último no es lógico ya que en todo caso no habría incrementos en el consumo de electricidad cuando la capacidad de consumo de electricidad se sature, ya que en las viviendas existe un número limitado de conectores y estos no pueden variar a menos que se realice una instalación. También consideremos que los hogares con mayor número de integrantes pueden corresponder con los de menores ingresos, por su bajo costo de oportunidad en ingreso ante un hijo adicional, y por tanto con los de menor consumo de electricidad al contar con menor poder adquisitivo y equipamiento de vivienda. Los signos de los coeficientes, que sugieren una tasa de crecimiento en consumo constante y una tasa de decremento variable al cambiar el número de integrantes del hogar, se deben a que existen costos de electricidad que se distribuyen entre los integrantes del hogar, como el servicio del refrigerador, de una bombilla de luz, el hecho de que una persona adicional mire la televisión no incrementa el consumo de electricidad, o que una persona entre a una habitación donde se hace uso de una bombilla eléctrica no incrementa el consumo de electricidad, se podría decir que algunos servicios de electricidad no son excluibles ni rivales en su uso. Otro aspecto por destacar es que la relevancia de estas variables cambia a lo largo de los deciles de consumo (ver 
gráficas 5 y 6) difiriendo así a las obtenidas por el estimador de MCO. En los deciles más altos al agregar un integrante se obtiene un crecimiento promedio menor y una tasa decreciente menor en el consumo de electricidad en comparación a los deciles bajos. Lo anterior podría estar relacionado con la base de consumo de estos hogares, ya que al contar con un mayor equipamiento en la vivienda como podría ser una caldera para la piscina, entonces el efecto de un integrante adicional no incrementaría tanto el consumo como la de los hogares con menor base de consumo de electricidad.

\section{Cuadro 2}

Elasticidades de la demanda en estudios $\operatorname{precios}^{1 /}$

\begin{tabular}{|c|c|c|c|}
\hline Autores & $\begin{array}{c}\text { Elasticidad } \\
\text { ingreso }\end{array}$ & $\begin{array}{c}\text { Elasticidad } \\
\text { precio }\end{array}$ & $\begin{array}{c}\text { Elasticidad } \\
\text { precio } \\
\text { cruzada }\end{array}$ \\
\hline Parti y Parti (1980) & 0.15 & -0.58 & - \\
\hline Dubin, Miedema y Chandran (1986) & 0.61 & -0.55 & - \\
\hline Baker, Blundell y Micklewright (1989) & 0.13 & -0.75 & - \\
\hline Baker (1992) & 0.24 & -0.62 & - \\
\hline Branch (1993) & 0.23 & -0.20 & - \\
\hline Reiss y White (2002) & 0.00 & -0.39 & - \\
\hline Fernández (2006) & $(0.14,0.58)$ & -0.59 & - \\
\hline Halvorsen y Larsen (2001) & $(0.06,0.13)$ & -0.44 & - \\
\hline Leth Petersen (2002) & 0.28 & - & - \\
\hline Medina y Vicéns (2012) & $(0.12,0.15)$ & - & - \\
\hline Dubin y McFadden (1984) & 0.02 & -0.26 & 0.39 \\
\hline Flippini y Pachauri (2004), invierno & 0.63 & -0.49 & -0.27 \\
\hline Flippini y Pachauri (2004), verano & 0.63 & -0.29 & 0.26 \\
\hline Flippini y Pachauri (2004), monzón & 0.60 & -0.51 & -0.65 \\
\hline Agostini, Plottier y Saavedra (2012) & $(0.11,0.12)$ & $(-0.38,-0.40)$ & $(0.14,0.16)$ \\
\hline Gutiérrez (2016) & $(0.04,0.10)^{*}$ & - & - \\
\hline Hancevic y Navajas (2015) & $(0.23,0.26)^{*}$ & - & - \\
\hline He y Reiner (2016), rural & $(0.33,0.38)$ & -3.59 & -2.03 \\
\hline He y Reiner (2016), urbano & $(0.33,0.46)$ & -2.91 & -2.36 \\
\hline Presente estudio & $(0.25,0.28)$ & $(-1.93,-1.42)$ & $(-1.81,-1.04)$ \\
\hline
\end{tabular}

$1 /$ Únicamente se incluyen resultados con significancia estadística. Los rangos indican la estimación puntual mínima y máxima para cada elasticidad.

* Para estos estudios, se trata de la elasticidad ingreso per cápita del hogar.

Nota: Por la diferencia de metodologías y datos, se recomienda acotar la comparabilidad de resultados al signo de los coeficientes para analizar el sentido económico del efecto.

Fuente: Fernández (2006), Medina y Vicéns (2011) y elaboración propia.

La proporción de bombillas sobre número de cuartos es una variable proxy para el tamaño de vivienda. De acuerdo con lo esperado los coeficientes estimados por MCO resulta con signo positivo indicando que a mayor tamaño de hogar mayor consumo de electricidad, mientras que las estimaciones por deciles de consumo resultan no ser estadísticamente distintas a la de MCO. 
Para el tipo de vivienda solo resultan significativas las variables ficticias de departamento y vecindad, las cuales de acuerdo con el estimador de MCO tienen una diferencia media de consumo de $-16.42 \%$ y $-21.24 \%$, respectivamente, en comparación con los hogares de familias que habitan en casas, mientras que las diferencias medias por decil de consumo resultaron no ser estadísticamente distintas a las de $\mathrm{MCO}^{27}$.

Además, los hogares con aire acondicionado en promedio consumen $60.22 \%$ más que los hogares que no tienen aire acondicionado, mientras que en las estimaciones por deciles resultaron entre $48.89 \%$ y $69.29 \%$, exhibiendo mayor magnitud para los deciles de alto consumo (gráfica 11). Se estima que los hogares con calefacción tienen un consumo promedio mayor en $18.86 \%$ respecto a los que no tienen calefacción, de acuerdo con el estimador de MCO, mientras que las estimaciones por deciles de consumo resultaron en un consumo promedio mayor de entre [13.89\%, $24.40 \%]$, aunque estas no son estadísticamente distintas a las de MCO. Al igual que Gutiérrez (2016), se encuentra que los hogares que hacen uso del aire acondicionado tienen un incremento en el consumo de electricidad considerable por lo que hacer un uso más eficiente de estos equipos puede contribuir de manera importante en el uso racional de la electricidad. En general, se podría buscar alternativas para hacer un uso más eficiente de estos equipos si se siguen las especificaciones del producto, ya que los quipos de enfriamiento están diseñados para trabajar bajo ciertas condiciones y estas se señalan en los manuales de usuario. Por ejemplo, la capacidad de enfriamiento de estos equipos está relacionada con su tamaño, entonces utilizar un aparato de aire acondicionado para tratar de enfriar una superficie superior para la cual fue diseñada provocará que el quipo opere en condiciones no óptimas y por tanto consuma mayor electricidad y reduzca su vida útil. Lo anterior también ocurre cuando la temperatura dentro de la vivienda es suficientemente alta como para que el equipo de enfriamiento logre alcanzar la temperatura solicitada, ya que esto haría que el equipo trabaje a su máxima capacidad durante su tiempo de uso. Por tanto, si los hogares complementaran con otras acciones el control de la temperatura de su vivienda y siguieran las especificaciones del producto podrían reducir el consumo en electricidad derivado del uso de equipos de enfriamiento. Sin embargo, los hogares podrían no estar tomando medidas para hacer un uso más eficiente de sus equipos de enfriamiento ya sea por asimetría de la información, falta de presupuesto, por la presencia del subsidio al consumo de electricidad durante la temporada de verano (que desincentiva el uso racional del recurso), etc.

Para los hogares que no cuentan con medidor de luz, se estima mediante MCO, que en promedio consumen $8.99 \%$ menos respecto a los hogares que sí cuentan

\footnotetext{
${ }^{27}$ Únicamente la estimación en el noveno decil de consumo de electricidad para la variable "vecindad" resulta estadísticamente distinta a la de MCO.
} 
con medidor de luz. Esto resulta contraintuitivo ya que se esperaría que al no medir su consumo de electricidad estos harían un uso irracional del recurso. Se debe considerar que el consumo de electricidad fue estimado de acuerdo con el gasto declarado por el hogar, es posible que los hogares que no tienen medidor de electricidad no estén pagando lo correspondiente a su consumo real y por tanto estemos subestimando su consumo. Las estimaciones obtenidas de la regresión cuantílica son únicamente significativas para los deciles de consumo de electricidad que van del primero al sexto, los cuales varían entre $5.81 \%$ y $-1.38 \%$. Se debe considerar que un hogar que declara altos niveles de gasto en electricidad debería contar con un medidor en su vivienda. Dado lo anterior, lo único razonable sería pensar que los hogares sin medidor de luz no están pagando lo que deberían por el servicio. Convendría estudiar el problema de falta de medición de consumo de electricidad en los hogares. Dado que los hogares incluidos en la muestra son aquellos que reportaron gasto en electricidad y que declararon que la luz eléctrica se obtiene del servicio público, estos usuarios sin medidor tendrían que ser usuarios de CFE.

De acuerdo con el estimador de MCO, los hogares con acceso a gas LP tienen una diferencia promedio en consumo de electricidad de $-3.38 \%$ respecto a los hogares con gas natural, mientras que las estimaciones de la regresión cuantílica no son estadísticamente distintas a las de MCO. Para los hogares que no tienen acceso a gas hay una diferencia promedio de consumo de $-28.99 \%$ respecto a los hogares que tienen acceso a gas natural, mientras que las estimaciones para cada decil de consumo oscilan entre $-38.53 \%$ y $-23.91 \%$. El resultado obtenido es contrario al esperado y se opone al conseguido por Hancevic y Navajas (2015) en el cual exponen que la falta de acceso a gas natural produce un mayor consumo de electricidad, aunque su análisis se limita al área metropolitana de Buenos Aires donde, de acuerdo con los autores, el $77.70 \%$ de los hogares están conectados a la red de gas natural. Dado que se esperaría que los hogares sin acceso a gas hagan uso de la electricidad para satisfacer su demanda de energía, y ya que la estimación sugiere que, como en la situación anterior, más bien disminuye el consumo de electricidad, entonces podría ser indicativo de que los hogares sin acceso a gas sean los más marginados y por tanto aquellos con menor equipamiento en su vivienda y menor consumo de electricidad. También existe la posibilidad de que su pago declarado de electricidad no refleje su consumo real, caso similar al de los hogares sin medidor de consumo. Según la encuesta nacional sobre consumo de energéticos en las viviendas particulares (ENCEVI) 2018, los combustibles más utilizados en las viviendas mexicanas son el gas LP, leña y gas natural con $79 \%, 11 \%$ y $7 \%$, respectivamente.

Contrario a lo esperado, el coeficiente para la variable "proporción de bombillas ahorradoras" resulta positivo tanto para la estimación de MCO como las estimaciones por deciles en la regresión cuantílica. Lo anterior puede 
justificarse como un "rebound effect" el cual consiste en un aumento en el consumo ante una mejora en eficiencia, en este caso los hogares que están ahorrando en iluminación destinan ese ahorro a otros servicios que requieren el uso de electricidad e inclusive incrementan su consumo. La relevancia de esta variable disminuye en los deciles más altos de consumo (gráfica 16). Esto indica que los programas públicos de renovación de equipos, como el de "Luz sustentable" y "Cambia tu viejo por uno nuevo" durante la gestión de Felipe Calderón (2006-2012), pueden incrementar el consumo de electricidad de los hogares a los que van dirigidos, por lo que dichos programas cumplen con un objetivo social, a través de un incremento de eficiencia en el uso de la electricidad de los hogares ${ }^{28}$.

Los coeficientes para las variables edad y edad al cuadrado de las estimaciones por deciles de consumo no resultaron ser estadísticamente distintas a las de MCO. Además, la magnitud de los coeficientes se aproxima a cero por lo que no resultan relevantes para fines de interpretación. Por otro lado, no se encuentra una diferencia media significativa de consumo entre hombre y mujer ni entre personas menores de 65 años y las de mayor o igual edad.

Se encuentra una diferencia media significativa entre los jefes de familia que concluyeron la secundaria, el bachillerato o la universidad en comparación con aquellos que no concluyeron secundaria. Para el estimador de MCO los jefes de familia que concluyeron sus estudios de secundaria, bachillerato y universidad tienen una diferencia media de consumo de electricidad de 3.68 $\%, 6.88 \%$ y $15.14 \%$, respectivamente, en comparación a los que no concluyeron la secundaria. En las estimaciones por deciles de consumo, las diferencias medias varían entre [2.26\%, $7.25 \%]$, [5.45 \%, $8.91 \%]$ y [13.55 $\%, 16.16 \%$, respectivamente, comparados con los jefes de familia que no concluyeron la secundaria. Para las estimaciones por deciles de consumo, únicamente la de la variable jefes de familia que concluyeron secundaria tiene diferencias promedio significativas respecto al estimador de MCO (gráfica 21). En particular la diferencia de consumo medio de electricidad de los jefes de familia con secundaria concluida respecto a los que no la concluyeron resulta ser no significativa en los deciles de consumo de electricidad más altos, contrario a los que concluyeron preparatoria y universidad que siguen teniendo diferencias cercanas al $5 \%$ y $14 \%$, respectivamente, respecto a los que no concluyeron secundaria.

Para las interacciones de estrato económico y población se observa que a mayor estrato económico mayor consumo de electricidad, así las diferencias medias estimadas por MCO para los estratos bajo en población rural, medio en

\footnotetext{
${ }^{28}$ El programa "Luz sustentable" consistía en obsequiar bombillas ahorradoras, mientras que el programa "Cambia tu viejo por uno nuevo" consistía en apoyos directos y créditos para renovación de electrodomésticos.
} 
población rural, bajo en población urbana, medio alto en población urbana y alto en población urbana resultaron en $-19.10 \%,-3.47 \%,-10.55 \%, 5.40 \%$ y $13.08 \%$, respecto al estrato medio en población urbana, mientras que para las estimaciones por decil de consumo estas oscilan entre [-26.56\%, -13.19\%], [$4.91 \%,-1.23 \%],[-19.42 \%,-7.58 \%],[1.69 \%, 9.61 \%]$ y [9.45\%, $14.96 \%]$, respectivamente. En general, estos resultados son intuitivos ya que se esperaría que las viviendas de los estratos más alto tengan mayor equipamiento del hogar.

Para considerar la influencia del clima en el consumo de electricidad se ha incorporado la temperatura promedio mensual en el modelo. De acuerdo con el estimador de MCO, se estima un incremento en el consumo de electricidad de $1.11 \%$ por grado centígrado adicional, mientras que las estimaciones por deciles de consumo fluctúan entre $0.78 \%$ y $1.28 \%$, aunque solo la correspondiente al cuarto decil de consumo resulta estadísticamente distinta a la obtenida en MCO, aunque solo excede ligeramente el límite inferior del intervalo de confianza de la estimación de MCO. Este resultado debe tomarse con reserva considerando las limitaciones expuestas anteriormente sobre esta variable. Las estimaciones de las diferencias promedio para el consumo de electricidad entre la Ciudad de México y el resto de las entidades federativas pueden consultarse en el anexo 7 y anexo 8 (gráficas 30-60).

Para el modelo estimado por MCO se obtiene una bondad del ajuste de 0.59. Debido a que la regresión cuantílica no tiene las propiedades del estimador MCO, no es posible calcular la $R^{2}$. Sin embargo, se presenta el estadístico de Pseudo- $R^{2}$ el cual se calcula como

$$
\text { Pseudo- } R^{2}=1-\frac{\sum_{i=1}^{i}\left\lceil y_{i}-\hat{y}_{i}\right\rceil}{\sum_{i=1}^{n}\left\lceil y_{i}-\hat{y}_{\theta}\right\rceil}
$$

y mide la bondad de ajuste para la regresión cuantílica (Vicéns y Sánchez, 2012).

\section{Cuadro 2}

Medida de bondad de ajuste de las estimaciones por decil

\begin{tabular}{cccccccccc}
\hline Decil & I & II & III & IV & V & VI & VII & VIII & IX \\
Pseudo- $R^{2}$ & 0.21 & 0.19 & 0.20 & 0.23 & 0.29 & 0.34 & 0.38 & 0.41 & 0.47 \\
\hline
\end{tabular}

Fuente: Elaboración propia con información de la ENIGH 2014, CFE y CRE

La bondad de ajuste es mayor para las estimaciones en los deciles más altos de consumo de electricidad. Estas son superiores a las obtenidas en el trabajo de Hancevic y Navajas (2015) las cuales no alcanzan el valor de 0.20. Otro trabajo 
que expone los cálculos de este estadístico es el trabajo de Medina y Vicéns (2011) cuyos valores resultan entre 0.41 y 0.43 .

\section{Conclusiones}

El objetivo de este trabajo es estimar la demanda de electricidad residencial en México mediante una regresión cuantílica. Por primera ocasión se utiliza información que permite identificar la tarifa de electricidad residencial a nivel municipal facilitando la conversión del gasto en electricidad a kilowatts-hora. Sin embargo, se incurre en una sobreestimación en el consumo de electricidad para los hogares que pagan derecho de alumbrado público ya que no se dispone de esta información.

El método de regresión cuantílica permite estimar rectas de regresión por cuantiles de la variable dependiente sin el inconveniente de ser considerablemente afectada por la presencia de heteroscedasticidad, datos atípicos o cambios estructurales ya que penaliza los errores de manera lineal y no de manera cuadrática como sucede con el estimador de MCO. De esta forma es posible analizar diferentes segmentos de la distribución como los hogares con consumo de electricidad más alto en donde se esperaría encontrar niveles elevados de ineficiencia energética.

Respecto a los resultados más relevantes, se encontró que la elasticidad ingreso de la demanda es inelástica, a través de los deciles de consumo, con un rango estimado de $[0.25,0.28]$, mientras que la elasticidad precio y precio cruzada de la demanda resultaron ser elásticas con rangos de [-1.93, -1.42] y [-1.81, $1.04]$, respectivamente.

Los resultados sugieren que los precios de los energéticos tienen mayor efecto en el consumo de la electricidad que el ingreso del hogar, y al igual que otros estudios, se encuentra que los cambios en los niveles de ingreso no tienen efectos importantes en el consumo de electricidad. Por otro lado, las estimaciones sugieren que los hogares son más sensibles ante cambios en precios de electricidad, en comparación a los cambios en ingreso, y que dicha relación es negativa y en particular de mayor magnitud para los hogares con mayores niveles de consumo de electricidad. Dado que en México no se focalizan los subsidios de electricidad por nivel de ingreso sino en función de la temperatura, el subsidio podría estar incentivando a los hogares a hacer un uso excesivo del recurso, por lo que convendría hacer un análisis más detallado sobre la determinación de tarifas residenciales y sus implicaciones en los patrones de consumo de los hogares, ya que podría ser una alternativa de política pública para corregir posibles fallos de mercado introducidos por medio de precios que no reflejen el costo marginal del servicio de electricidad. 
Igualmente se recomienda tener cautela con el resultado obtenido para la elasticidad precio-cruzada de la demanda ya que se pensaría que hay un grado de sustitución entre energéticos, mientras que la estimación sugiere que se tratan de bienes complementarios.

Se recomienda, para futuras investigaciones, un análisis profundo que explore vertientes como los determinantes de las inversiones de los hogares en el equipamiento de su vivienda, la capacidad de respuesta de los hogares ante cambios en precios de energéticos en sus asignaciones de equipamiento de vivienda, etc. Igualmente se considera que este resultado podría derivarse de errores de medición o de muestra.

También se observa que los hogares, equipados con aire acondicionado, consumen en promedio entre $69 \%$ y $49 \%$ más que el resto de los hogares, por lo que hacer un uso más eficiente de estos equipos puede contribuir de manera importante en el uso racional de la electricidad. Existe la posibilidad de que los hogares no estén haciendo un uso eficiente de sus equipos de enfriamiento ya sea por asimetría de la información, falta de presupuesto, por la presencia del subsidio al consumo de electricidad durante la temporada de verano (que desincentiva el uso racional del recurso), etc.

Además, contrario a lo esperado, se encuentra que los hogares sin medidor consumen menos electricidad que los hogares con medidor. Lo anterior levanta la sospecha de que el gasto de los hogares sin medidor no refleje el consumo de kwh y por tanto se haya subestimado el consumo de estos hogares, ya que este se calculó a partir del gasto en electricidad declarado por los hogares. Es por esto por lo que se considera que se debería estudiar el problema de la falta de medición de consumo de electricidad en los hogares.

Otro resultado contraintuitivo fue el obtenido para los hogares sin acceso a gas, ya que se encuentra que estos consumen menor electricidad cuando se esperaría que estos hogares hagan uso de la electricidad para satisfacer su demanda de energía. Lo anterior levanta dos sospechas: 1) que los hogares sin acceso a gas sean los más marginados y por tanto aquellos con menor equipamiento en su vivienda y menor consumo de electricidad, y 2) que su pago declarado de electricidad no refleje su consumo real.

Los resultados expuestos en este artículo no deben ser generalizados para todo el año ya que por el periodo de levantamiento de la ENIGH estos representan el consumo de electricidad durante el tercer trimestre del año, y coincide con la temporada de verano, que es la de mayor consumo. 


\section{Referencias}

[1] Agostini, C., Plottier, C. y Saavedra, E. (2012). "La demanda residencial por energía eléctrica en Chile”, Journal Economía Chilena (The Chilean Economy), Central Bank of Chile, vol. 15(3), 64-83.

[2] Baker, P., y Blundell, R. (1991), The Microeconometric Approach to Modelling Energy Demand: Some Results for UK Households, Oxford Review of Economic Policy, 7, issue 2, p. 54-76.

[3] Baker, P., Blundell, R. y Micklewright, J. (1989) "Modelling household energy expenditures using microdata", The Economic Journal, 99, September, 720-738.

[4] Branch, R. (1993). "Short run income elasticity of demand for residential electricity using consumer expenditure survey data", The Energy Journal, 14 (4), 111 121. SLEP']

[5] Bound, J., Jaeger, D. y Baker, R. (1995) "Problems with instrumental variables when the correlation between the instruments and the endogenous explanatory variables is weak". Journal of the American Statistical Association. Applications and case studies, 90(430), 443-450.

[6] Comisión Federal de Electricidad. Tarifas para el suministro y venta de energía eléctrica (2014): URL < https://app.cfe.mx> [último acceso: Agosto de 2017].

[7] Comisión Reguladora de Energía: URL <http://www.calculadora.cre.gob.mx> [último acceso: Agosto de 2017].

[8] Cuadrado, J. (2010). "Política Económica: elaboración, objetivo e instrumentos". McGraw Hill.

[9] Dubin, J. y McFadden, D. (1984) “An econometric analysis of residential electric appliance holdings and consumption" Econometrica, 52(2), 345-362.

[10] Dubin, J., Miedema, A. y Chandran, R. (1986): Price Effects of Energy-Efficient Technologies: A Study of Residential Demand for Heating and Cooling.

[11] Fernández, L. (2006). "La demanda residencial de electricidad en España: Un análisis microeconométrico", Tesis doctoral, Universidad de Barcelona: URL <http://diposit.ub.edu/dspace/handle/2445/42612> [último acceso:

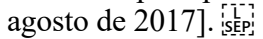

[12] Gutiérrez, A. (2016).“Determinantes de consumo eficiente de energía eléctrica en el sector residencial en México: Un enfoque de regresión cuantílica", Tesina de maestría, Centro de Investigación y Docencia Económica, A.C.,

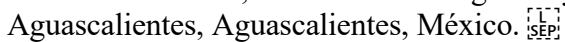

[13] Halvorsen, B. y Larsen, B. (2001). "The flexibility of household electricity demand over time" en Resource and Energy Economics, 23(1), pp. 1-18.

[14] Hancevik, P. y Navajas, F. (2015). "Consumo Residencial de Electricidad y Eficiencia Energética”, El Trimestre Económico, LXXXII (4), No.328, 897-927. ín

[15] He, X. y Reiner, D. (2016). "Electricity Demand and Basic Needs: Empirical Evidence from China's Households", Energy Policy, Elsevier, 90(C), 212221.

[16] Instituto Nacional de Estadística y Geografía (2015). Encuesta Nacional de Ingresos y Gastos de los Hogares, 2014.

[17] Heckman, J.J. (1979). "Sample selection bias as a specification error", Econometrica, 47(1), 153-161. 
[18] Koenker, R. \& Bassett, G. (1978). "Regression Quantiles”, Econometrica, 46(1), 33-50.

[19] Leth-Petersen, S. (2002). "Micro econometric modelling of household energy use: testing for dependence between demand for electricity and natural gas" en Energy Journal, 23(4), pp. 57-84.

[20] Medina, E. y Vicéns, J. (2011). "Factores determinantes de la demanda eléctrica de los hogares en España: Una aproximación mediante regresión cuantílica", Estudios de Economía Aplicada, 29(2), 515-538.

[21] Nuño, J. (2014). "El Cobro del Alumbrado Público (DAP), en los Municipios", Hacienda Municipal, Instituto para el Desarrollo Técnico de las Haciendas Públicas, Revista trimestral, No. 121, 38-44. [s-

[22] Pachauri, S. y Filippini, M. (2004). "Elasticities of electricity demand in urban

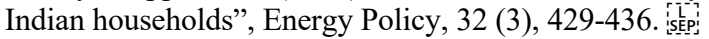

[23] Parti and Parti (1980). The Total and Appliance-Specific Conditional Demand for Electricity in the Household Sector, Bell Journal of Economics, 11, issue 1, p. 309-321.

[24] Reiss, P. and White, M. (2005), Household Electricity Demand, Revisited, Review of Economic Studies, 72, issue 3, p. 853-883.

[25] Sánchez, B., y Vicéns, J. (2012). "Regresión cuantílica: Estimación y contrastes", Instituto L.R. Klein-Centro Gauss. U.A.M D.T. no. 21.

[26] Shin, J. (1985). "Perception of price when price information is costly: evidence from residential electricity demand", The Review of Economics and

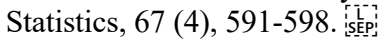

[27] Tinbergen, J. (1959). Problems of planning economic policy. International Social Science Journal. XI, 3, p. 351 - 360. 